\title{
Isotropic Seebeck coefficient of aligned single-wall carbon nanotube films
}

\author{
Kengo Fukuhara, ${ }^{1}$ Yota Ichinose, ${ }^{1}$ Hiroyuki Nishidome, ${ }^{1}$ Yohei Yomogida, ${ }^{1}$ \\ Fumiya Katsutani, ${ }^{2}$ Natsumi Komatsu, ${ }^{2}$ Weilu Gao, ${ }^{2}$ Junichiro Kono, ${ }^{2,3,4}$ \\ and Kazuhiro Yanagi ${ }^{1, a)}$ \\ ${ }^{1}$ Department of Physics, Tokyo Metropolitan University, Tokyo 192-0397, Japan \\ ${ }^{2}$ Department of Electrical and Computer Engineering, Rice University, Houston, Texas 77005, USA \\ ${ }^{3}$ Department of Physics and Astronomy, Rice University, Houston, Texas 77005, USA \\ ${ }^{4}$ Department of Materials Science and NanoEngineering, Rice University, Houston, Texas 77005, USA
}

(Received 11 October 2018; accepted 27 November 2018; published online 13 December 2018)

\begin{abstract}
How the morphology of a macroscopic assembly of nanoobjects affects its properties is a longstanding question in nanomaterials science and engineering. Here, we examine how the thermoelectric properties of a flexible thin film of carbon nanotubes depend on macroscopic nanotube alignment. Specifically, we have investigated the anisotropy of the Seebeck coefficient of aligned and gated single-wall carbon nanotube thin films. We varied the Fermi level in a wide range, covering both the $p$-type and $n$-type regimes, using electrolyte gating. While we found the electrical conductivity along the nanotube alignment direction to be several times larger than that in the perpendicular direction, the Seebeck coefficient was found to be fully isotropic, irrespective of the Fermi level position. We provide an explanation for this striking difference in anisotropy between the conductivity and the Seebeck coefficient using Mott's theory of hopping conduction. Our experimental evidence for an isotropic Seebeck coefficient in an anisotropic nanotube assembly suggests a route toward controlling the thermoelectric performance of carbon nanotube thin films through morphology control. Published by AIP Publishing. https://doi.org/10.1063/1.5066021
\end{abstract}

There is an increasing demand for high-performance flexible thermoelectric materials for their compatibility with various shaped objects and batteries for growing flexible thin film electronics and sensors. As flexible thermoelectric materials, polymers and carbon nanotubes are strong candidates, ${ }^{1}$ and single wall carbon nanotubes (SWCNTs) are particularly attractive because their one-dimensionality, such as their van Hove singularities in electronic density of states, is expected to have superior thermoelectric performance. ${ }^{2}$ SWCNTs prepared in the form of flexible, randomly oriented thin films have exhibited relatively large Seebeck coefficients $\left(150-300 \mu \mathrm{VK}^{-1}\right)$ in semiconducting type samples. ${ }^{3-5}$ In addition, they can be readily made $p$-type or $n$-type through Fermi-level control by chemical or electro-chemical approaches, ${ }^{4,6,7}$ suitable for large scale $p-n$ thermoelectric flexible devices. ${ }^{6}$ Thus, there are currently worldwide efforts to understand, control, and improve the thermoelectric properties of SWCNT thin films.

Thermoelectric properties of SWCNT films are strongly influenced by many factors such as their electronic type (metallic versus semiconducting), ${ }^{3-5}$ the location of the Fermi level $\left(E_{\mathrm{F}}\right),{ }^{4,6,7}$ and sample morphology. ${ }^{8-10}$ For improvement of thermoelectric performance of SWCNT films, it is necessary to control and optimize the thermoelectric parameters of the sample, that is, Seebeck coefficient $S$, electrical conductivity $\sigma$, and thermal conductivity $\kappa$. For optimum power generation from a given temperature gradient, one needs to maximize the value of $S^{2} \sigma$, known as the power factor. Previously, it has been revealed that preparation of highpurity semiconducting SWCNT films is important to achieve large Seebeck coefficients. ${ }^{3-5}$ Also, it has been shown that

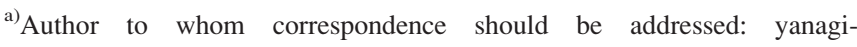
kazuhiro@tmu.ac.jp.
}

precise $E_{\mathrm{F}}$ tuning is crucial for enhancing the power factor of SWCNT films. ${ }^{4}$ However, these previous studies were made on samples in which SWCNTs were randomly oriented. Several studies have reported an enhancement of the power factor through an increase in the conductivity induced by a change in morphology. ${ }^{8-10}$ However, an understanding of how morphology affects thermoelectric properties remains elusive.

To tackle this problem, a systematic study of thermoelectric properties of aligned SWCNT thin films is desired. Such a study should allow us to examine the relationships between geometrical nanotube arrangements and thermoelectric properties. However, to date, how $S$ depends on the nanotube alignment has not been well understood. A previous report indicated that the value and the anisotropy of $S$ change with sample annealing, ${ }^{11}$ but it is important to note that both $\sigma$ and $S$ sensitively depend on $E_{\mathrm{F}}{ }^{4,12}$ Hence, to provide an unambiguous understanding of how $S$ changes with nanotube alignment, it is crucial to perform systematic measurements of the $E_{\mathrm{F}}$ dependence of $S$ in a well-aligned SWCNT film in both parallel and perpendicular directions in a wide range of $E_{\mathrm{F}}$ values.

Here, we report a systematic and detailed study on the values and anisotropy of the electrical conductivity and the Seebeck coefficient in films of aligned SWCNTs as a function of $E_{\mathrm{F}}$. We employed the recently reported controlled vacuum filtration method ${ }^{13}$ to fabricate wafer-scale singledomain thin films of aligned SWCNTs. By using the electrolyte gating method, ${ }^{4,12,14}$ we systematically varied the $E_{\mathrm{F}}$ of the SWCNT thin film in a wide range, observing a sign change in $S$ when the system goes from the $p$-type region to the $n$-type region. The value of $\sigma$ showed strong in-plane anisotropy, i.e., more conducting in the direction parallel to 
the nanotube alignment direction. However, surprisingly, $S$ did not display any sign of anisotropy, irrespective of the position of $E_{\mathrm{F}}$, which agrees well with what we expect from Mott's hopping theory.

We suspended SWCNTs with an average diameter of $1.4 \mathrm{~nm}$ in an aqueous solution of $2 \%$ sodium deoxycholate (DOC). The SWCNTs were a mixture of metallic and semiconducting nanotubes, produced by the arc discharge method (Meijo Nano Carbon Co., ARC SO). We diluted the suspension to $0.02 \%$ DOC, which we then used to fabricate aligned films using the controlled vacuum filtration method described in Ref. 13. We measured $\sigma$ and $S$ of these aligned films in directions that are parallel and perpendicular to the nanotube alignment direction [Fig. 1(a)]. We used polarization-dependent Raman measurements [Fig. 1(b)] to determine the nanotube alignment direction and evaluate the alignment degree (see Fig. S1 in the supplementary material). When we define $I_{\|}$and $I_{\perp}$ as Raman intensities for pump polarization parallel and perpendicular to the nanotube alignment direction, respectively, the typical $I_{\|} / I_{\perp}$ values in our films were around $3.5 \pm 1.0$.

We first measured the $\sigma$ and $S$ values in the directions parallel and perpendicular to the alignment direction without applying a gate voltage. Figure 1(c) shows a photograph of the experimental setup used for these measurements. We cut the aligned film into a $5 \mathrm{~mm} \times 5 \mathrm{~mm}$ square (thickness: $35 \mathrm{~nm}$ ); we define the $x$-direction ( $y$-direction) to be the parallel (perpendicular) direction. Thermocouples and heaters were attached by silver paste to the film to measure $\sigma$ and $S$ in both the $x$ - and $y$-directions. The details of the experimental setup are described in the supplementary material. We summarize the obtained results in Table I. As is shown here, $\sigma$ along the parallel direction was three times larger than that along the perpendicular direction. However, the measured values of $S$ in both directions were $29 \mu \mathrm{V} / \mathrm{K}$; that is, the Seebeck coefficient is isotropic within experimental errors.

However, as mentioned above, the value of $S$ strongly depends on the location of the Fermi level. Therefore, we next investigated how $\sigma$ and $S$ change with the gate voltage. Figure 2 schematically shows the experimental setup used, which is essentially the same as those used in previous studies. ${ }^{4}$ Source, drain, reference, and gate electrodes were formed on polyimide substrates covered by a $10-\mu \mathrm{m}$-thick parylene layer. Electron or hole injection was made through electric double layer formation using ionic liquid [N,N,N-trimethyl-N-propylammonium bis(trifluoromethanesulfonyl)imide (TMPA-TFSI), Kanto Kagaku Co.], and the amount of injected carriers was controlled by the applied gate voltage. The aligned SWCNT film was placed over the source and drain electrodes as a channel. We prepared two devices: one had a source-drain channel parallel to the SWCNT alignment direction, whereas the other had a source-drain channel perpendicular to the alignment direction. The details of experimental setups are described in the supplementary material.

Figure 3(a) shows the measured $\sigma$ values along the parallel and perpendicular directions as a function of gate voltage. It is clearly seen that the $\sigma$ value along the parallel direction was higher than that along the perpendicular direction in the whole range of gate voltages (see Fig. S2 of the supplementary material). The value along the parallel

(a)

\section{Parallel}

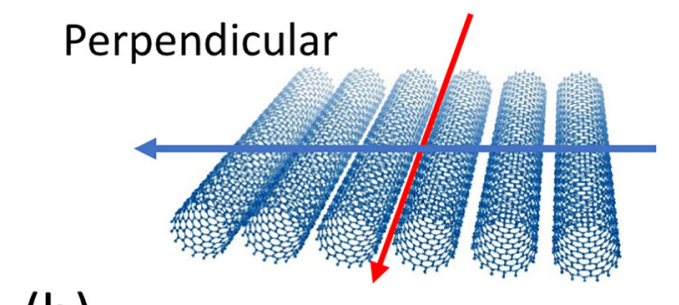

(b)
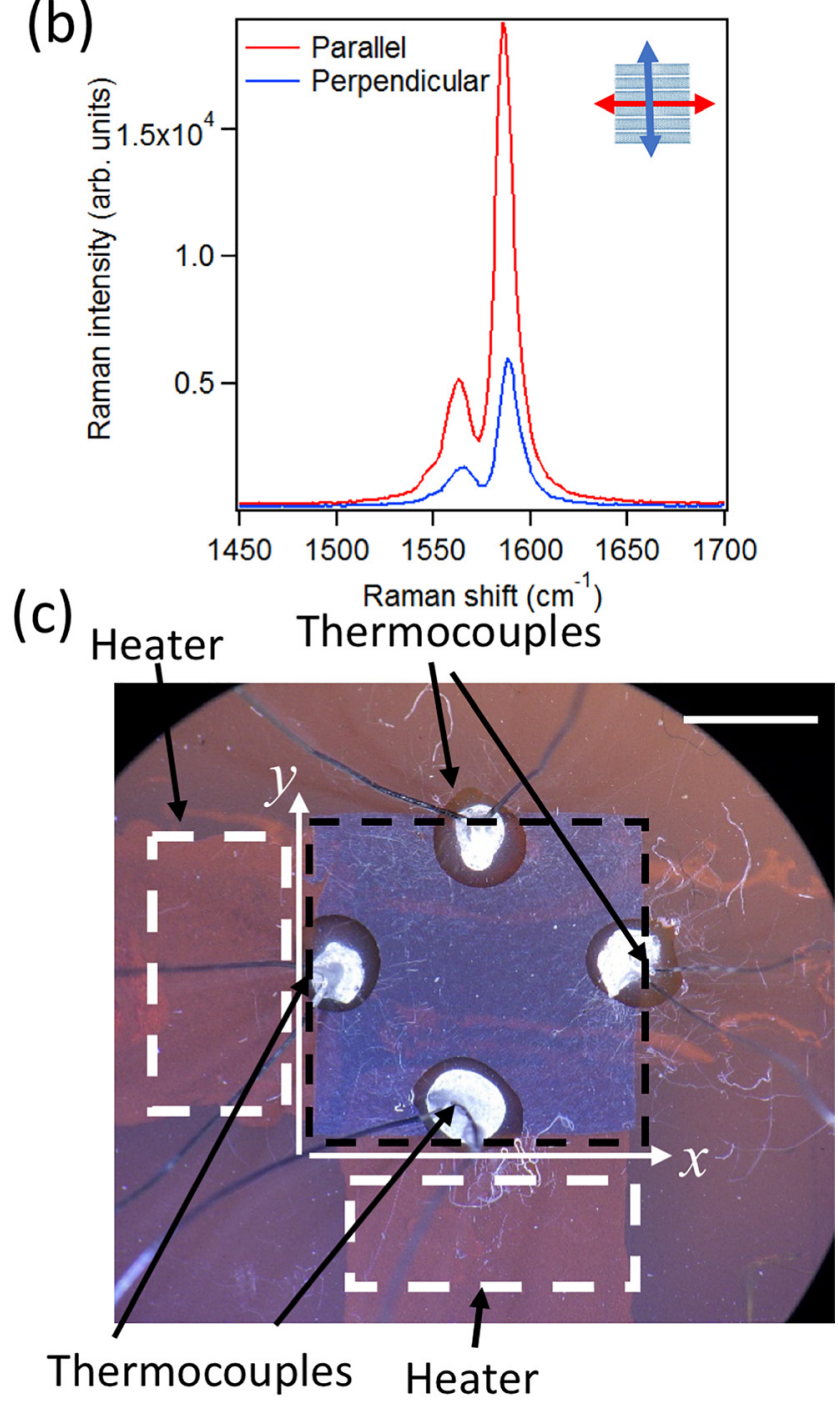

FIG. 1. (a) Schematic illustration of the parallel and perpendicular directions in an aligned single-wall carbon nanotube film. (b) Typical Raman spectra taken with excitation light polarized parallel and perpendicular to the aligned axis. (c) A photograph of a fabricated device for directiondependent electrical conductivity and Seebeck coefficient measurements. Heaters (white dashed lines) were attached on the rear side of the substrate. The black dashed line indicates the region where an aligned SWCNT film was transferred. The alignment axis was set to be parallel to the $x$-direction. The scale bar is $2 \mathrm{~mm}$.

TABLE I. Electrical conductivity and Seebeck coefficient values obtained in the parallel and perpendicular directions in an aligned SWCNT thin film without applying a gate voltage.

\begin{tabular}{lcc}
\hline & Electrical conductivity $\left(\mathrm{S} \mathrm{m}^{-1}\right)$ & Seebeck coefficient $\left(\mu \mathrm{V} \mathrm{K}^{-1}\right)$ \\
\hline Parallel & $9.7 \times 10^{4}$ & 29 \\
Perpendicular & $3.5 \times 10^{4}$ & 29 \\
\hline \hline
\end{tabular}




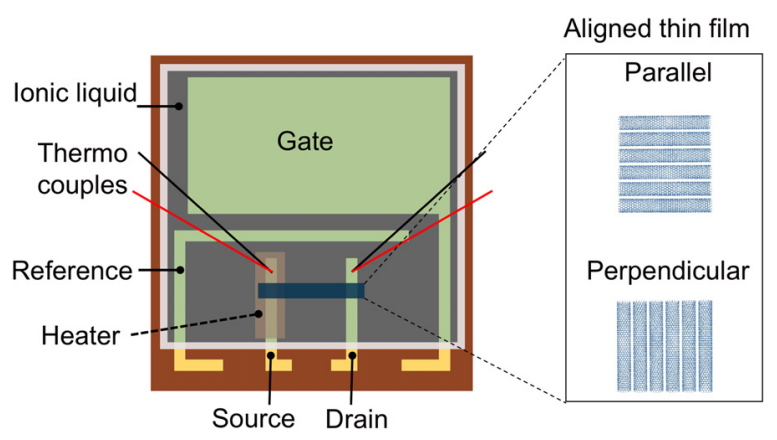

FIG. 2. Schematic illustration of a device for measuring the conductivity and the Seebeck coefficient in the parallel and perpendicular directions in an aligned SWCNT thin film as a function of gate voltage.
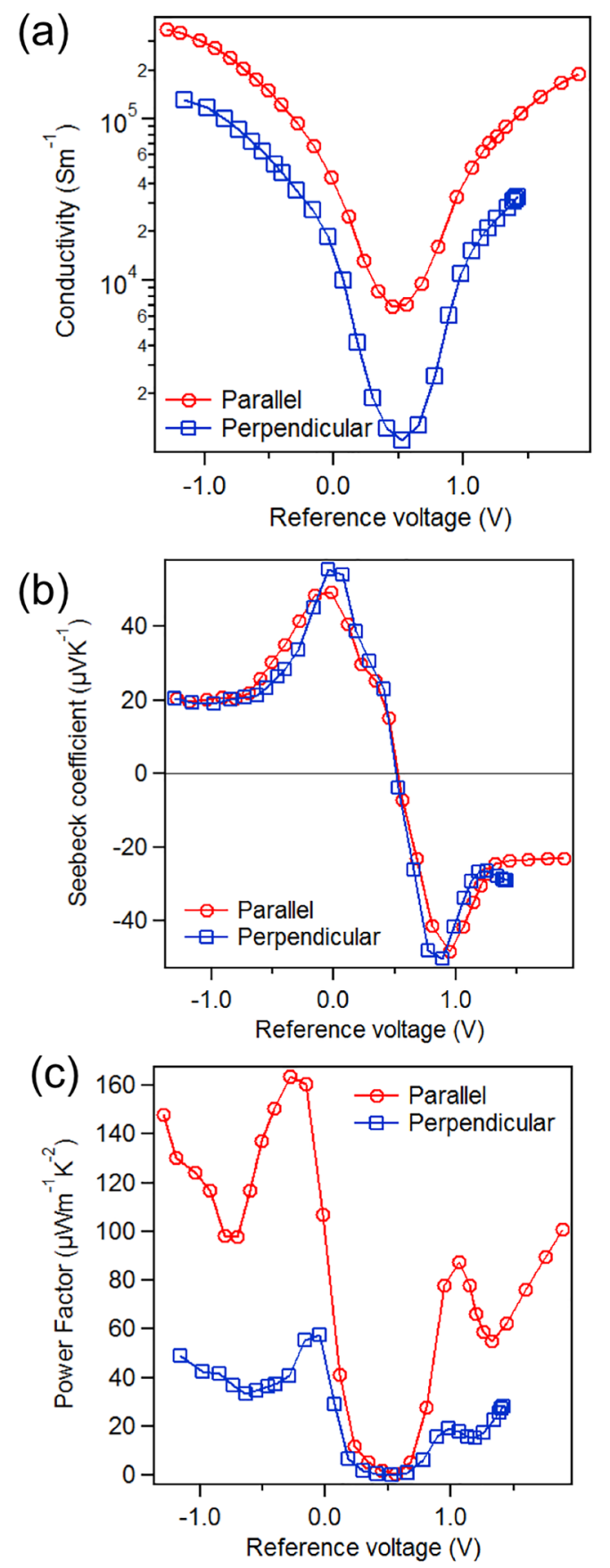

FIG. 3. (a) Conductivity, (b) Seebeck coefficient, and (c) power factor as a function of gate voltage in the parallel (red open circles) and perpendicular (blue open squares) directions in an aligned SWCNT thin film. direction was almost 7 times larger than that in the perpendicular direction around the charge-neutrality point, and the ratio of conductivity of parallel to perpendicular directions tends to decrease with the shift of gate voltage and reached around 2.5-3 as shown in Fig. S2 of the supplementary material. However, the measured $S$ values exhibited a completely isotropic behavior. Figure 3(b) shows $S$ as a function of gate voltage. In both directions, the sign of $S$ changed when the charge type of the system changes between $p$-type and $n$ type. The peak values of $S$ in the $p$ - and $n$-regions were approximately $50 \mu \mathrm{V} \mathrm{K}^{-1}$ and $-50 \mu \mathrm{V} \mathrm{K}^{-1}$, respectively. In stark contrast to $\sigma$, the $S$ values were the same between the parallel and perpendicular directions, within experimental errors, at all gate voltage values. Combining the results obtained for $\sigma$ and $S$, Fig. 3(c) plots the power factor, $S^{2} \sigma$, against gate voltage. The anisotropy of $S^{2} \sigma$ seen here simply reflects the anisotropy of $\sigma$.

To shed light on these observed characteristics of $\sigma$ and $S$, we use Mott's theory of hopping conduction, ${ }^{15}$ which has described electrical conduction phenomena in heterogeneous films containing metallic and semiconducting SWCNTs. ${ }^{16}$ According to Mott, the electrical conductivity and the Seebeck coefficient can be written as

$$
\begin{gathered}
\sigma=-\int \sigma_{E} \frac{\partial f}{\partial E} d E, \\
S=-\frac{k}{e} \frac{\int \sigma_{E} \frac{E-E_{F}}{k T} \frac{\partial f}{\partial E} d E}{\int \sigma_{E} \frac{\partial f}{\partial E} d E} .
\end{gathered}
$$

Here, $\sigma_{E}=e^{2} p R^{2} N\left(E_{F}\right), N\left(E_{F}\right)$ is the carrier density at the Fermi level, $f$ is the Fermi distribution function, $p$ is the probability per unit time that an electron jumps from one site to another site, and $R$ is related to the mean distance between the hopping sites. ${ }^{15}$ We can assume that the value of $\sigma_{E}$ will change depending on the morphology of the SWCNT network. In an aligned SWCNT thin film, the $\sigma_{E}$ value should be different between the parallel and perpendicular directions. For example, we can assume that $R$ in the perpendicular direction should be short because of the short scattering time compared to that in the parallel direction. As a result, $\sigma_{E}$ in the perpendicular direction becomes smaller than that in the parallel direction, which results in conductivity anisotropy through Eq. (1), as observed experimentally. However, in the case of $S, \sigma_{E}$ appears both in the numerator and in the denominator of the expression, as shown in Eq. (2). Thus, the anisotropy of $R$ (and hence $\sigma_{E}$ ) cancels, leading to an isotropic $S$, consistent with our observations. The details are described in the supplementary material.

In conclusion, we clarified the conductivities, Seebeck coefficients, and power factors of aligned SWCNT films for directions both parallel and perpendicular to the SWCNT alignment direction. We controlled the position of the Fermi level in a wide range, covering both the $p$-type regime and the $n$-type regime. We found that the electrical conductivity value in the parallel direction is much higher than that in the perpendicular direction. However, we found that the Seebeck coefficient is isotropic, i.e., its value is the same in 
both the parallel and perpendicular directions, regardless of the position of the Fermi level. These data firmly established the isotropic character of Seebeck coefficients in aligned SWCNT films. Although the results are consistent with what we expect from Mott's formulas for the electrical conductivity and the Seebeck coefficient, more microscopic models are needed to quantitatively understand our observations. Technologically, our discovery of simultaneously anisotropic $\sigma$ and isotropic $S$ in an aligned SWCNT film implies that their ratio is continuously tunable, which is potentially useful for managing charge and thermal flows in flexible thermoelectric devices through morphology engineering.

See supplementary material for a picture of a typical SWCNT aligned film, the ratio of parallel to perpendicular conductivities as a function of reference voltage, details of experimental setups for thermoelectric measurements, and discussion on isotropic characteristics of the Seebeck coefficient based on Mott's hopping theory.

K.Y. acknowledges support of JSPS KAKENHI, Grant Nos. JP17H06124, JP17H01064, JP18H01816, and JST CREST through Grant No. JPMJCR17I5, Japan. F.K., N.K., W.G., and J.K. acknowledge support of the Department of Energy Basic Energy Sciences through Grant No. DEFG0206ER46308 (optical spectroscopy), the National Science Foundation through Award No. ECCS-1708315 (device fabrication), and the Robert A. Welch Foundation through Grant No. C-1509 (sample preparation).
${ }^{1}$ O. Bubnova and X. Crispin, Energy Environ. Sci. 5, 9345 (2012).

${ }^{2}$ L. D. Hicks and M. S. Dresselhaus, Phys. Rev. B 47, 16631 (1993).

${ }^{3}$ Y. Nakai, K. Honda, K. Yanagi, H. Kataura, T. Kato, T. Yamamoto, and Y. Maniwa, Appl. Phys. Express 7, 025103 (2014).

${ }^{4}$ K. Yanagi, S. Kanda, Y. Oshima, Y. Kitamura, H. Kawai, T. Yamamoto, T. Takenobu, Y. Nakai, and Y. Maniwa, Nano Lett. 14, 6437 (2014).

${ }^{5}$ A. D. Avery, B. H. Zhou, J. Lee, E. Lee, E. M. Miller, R. Ihly, D. Wesenberg, K. S. Mistry, S. L. Guillot, B. L. Zink, Y. Kim, J. L. Blackburn, and A. J. Ferguson, Nat. Energy 1, 16033 (2016).

${ }^{6}$ Y. Nonoguchi, K. Ohashi, R. Kanazawa, K. Ashiba, K. Hata, T. Nakagawa, C. Adachi, T. Tanase, and T. Kawai, Sci. Rep. 3, 3344 (2013).

${ }^{7}$ T. Fukumaru, T. Fujigaya, and N. Nakashima, Sci. Rep. 5, 7951 (2015).

${ }^{8}$ W. Zhou, Q. Fan, Q. Zhang, K. Li, L. Cai, X. Gu, F. Yang, N. Zhang, Z. Xiao, H. Chen, S. Xiao, Y. Wang, H. Liu, W. Zhou, and S. Xie, Small 12, 3407 (2016).

${ }^{9}$ W. Zhou, Q. Fan, Q. Zhang, L. Cai, K. Li, X. Gu, F. Yang, N. Zhang, Y. Wang, H. Liu, W. Zhou, and S. Xie, Nat. Commun. 8, 14886 (2017).

${ }^{10}$ B. A. MacLeod, N. J. Stanton, I. E. Gould, D. Wesenberg, R. Ihly, Z. R. Owczarczyk, K. E. Hurst, C. S. Fewox, C. N. Folmar, K. H. Hughes, B. L. Zink, J. L. Blackburn, and A. J. Ferguson, Energy Environ. Sci. 10, 2168 (2017).

${ }^{11}$ J. Hone, M. C. Llaguno, N. M. Nemes, A. T. Johnson, J. E. Fischer, D. A. Walters, M. J. Casavant, J. Schmidt, and R. E. Smalley, Appl. Phys. Lett. 77, 666 (2000).

${ }^{12}$ Y. Oshima, Y. Kitamura, Y. Maniwa, and K. Yanagi, Appl. Phys. Lett. 107, 043106 (2015).

${ }^{13}$ X. He, W. Gao, L. Xie, B. Li, Q. Zhang, S. Lei, J. M. Robinson, E. H. Haroz, S. K. Doorn, W. Wang, R. Vajtai, P. M. Ajayan, W. W. Adams, R. H. Hauge, and J. Kono, Nat. Nanotechnol. 11, 633 (2016).

${ }^{14}$ K. Yanagi, R. Okada, Y. Ichinose, Y. Yomogida, F. Katsutani, W. Gao, and J. Kono, Nat. Commun. 9, 1121 (2018).

${ }^{15}$ N. F. Mott and E. A. Davis, Electronic Processes in Non-crystalline Materials (Oxford University Press, 1979).

${ }^{16}$ K. Yanagi, H. Udoguchi, S. Sagitani, Y. Oshima, T. Takenobu, H. Kataura, T. Ishida, K. Matsuda, and Y. Maniwa, ACS Nano 4, 4027 (2010). 